\title{
Identification of Magnetic Phases in Natural Ochres by Mössbauer Spectroscopy
}

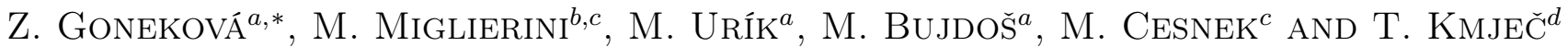 \\ ${ }^{a}$ Institute of Laboratory Research on Geomaterials, Comenius University, \\ Mlynská dolina, 84215 Bratislava, Slovakia
}

${ }^{b}$ Slovak University of Technology in Bratislava, Faculty of Electrical Engineering and Information Technology, Institute of Nuclear and Physical Engineering, Ilkovičova 3, 81219 Bratislava, Slovakia

${ }^{c}$ Department of Nuclear Reactors, Faculty of Nuclear Sciences and Physical Engineering,

Czech Technical University in Prague, V Holešovičkách 2, 18000 Prague 8, Czech Republic

${ }^{d}$ Department of Low Temperature Physics, Faculty of Mathematics and Physics, Charles University, V Holešovičkách 2, 18000 Prague 8, Czech Republic

\begin{abstract}
The Mössbauer spectroscopy is one of the few analytical techniques that provide information on the investigated system both from the viewpoint of its structural arrangement and also magnetic order. Here, we demonstrate how ${ }^{57}$ Fe Mössbauer spectroscopy can contribute to identification of magnetic phases in rather ordinary Fe-containing materials like natural ochres. Fe-ochre precipitates were collected at an abandoned antimony deposit in East Slovakia. Three sets of samples were studied: (i) as-collected, (ii) ochres sterilized at $95{ }^{\circ} \mathrm{C}$ for 30 min, and (iii) Fe-ochre precipitates which were exposed to fungal bioleaching after sterilization. The Mössbauer spectra from all three batches were recorded at 300, 80, and $5 \mathrm{~K}$. Presence of magnetic phases was revealed only at the lowest temperature. At higher temperatures, all samples show significant relaxation of magnetic moments which indicates that the crystalline grains are very small and exhibit superparamagnetic behaviour.
\end{abstract}

DOI: 10.12693/APhysPolA.137.667

PACS/topics: Mössbauer spectroscopy, Fe ochre, fungi, bioleaching

\section{Introduction}

Ferric ochres are the part of the geochemical barriers that effectively take up hazardous substances in the environment [1]. Complete mineralogical and specific characterization of them is difficult because Fe-ochres are complex heterogeneous mixtures of different minerals [2]. The identification of the mineral iron phases and their oxidation states can assist in assessing the geochemical barrier composition in which the individual mineral phases exhibit different absorption capacity [3].

Filamentous fungi contribute significantly to the mobility of elements that are potentially dangerous to the environment. They produce strongly acidic or chelating compounds, such as polycarboxylic organic acids, being able to solubilise metals or metalloids from different oxidized ferric minerals or amorphous phases by redox processes or by $\mathrm{pH}$ lowering. However, the released metals or metalloids can be subsequently accumulated and metabolized by microorganisms [4]. The Mössbauer spectrometry is a suitable analytical method for identifying the structure in the natural mineral phases of iron, determining the iron oxidation states, chemical bonds, magnetic, and electrical interactions [5].

As this method has been successfully applied by our research group previously to evaluate microbially induced

*corresponding author; e-mail: gonekova4@uniba.sk changes in synthetic ferric phases [6], consequently, the analysis of microbial effects on significantly more complex and structurally challenging natural ferric material is addressed in this paper.

\section{Experimental details}

Natural Fe-containing ochres were collected at the outflow from the Agnes mine located in Poproč (Slovakia). The obtained samples were supplied as suspensions, then decanted, filtered, and finally dried. Using these procedures, the first, the so-called as-collected, sample (i) was obtained. Sterilization of the sample (i) at $95^{\circ} \mathrm{C}$ for $30 \mathrm{~min}$ resulted in the second sample (ii). Samples were sterilized in order to ensure optimal conditions for the selected microorganism during the study of bioleaching processes. Subsequently, static cultivation with filamentous fungus Aspergillus niger (incubation time: 14 days at $300 \mathrm{~K}, 0.1 \mathrm{~g}$ of the sample (ii) in $45 \mathrm{ml}$ nutrient medium (HiMedia, India) inoculated with $5 \mathrm{ml}$ of fungal spore suspension) has formed the third sample (iii).

The Mössbauer effect experiments were performed in transmission geometry using a standard spectrometer equipped with a ${ }^{57} \mathrm{Co} / \mathrm{Rh}$ source. The spectra were collected at 300,80 , and $5 \mathrm{~K}$. Calibration of the velocity scale was performed by an $\alpha$-Fe foil. The isomer shift values are given relative to the centre of its spectrum at room temperature. The Mössbauer data were analysed using the Confit program [7]. 


\section{Results and discussion}

Typical Mössbauer spectrum measured at room temperature is demonstrated in Fig. 1a where sample (iii) is shown as an example. All three samples show the same doublet-like features at $300 \mathrm{~K}$. The crystalline phases are likely to be very small as they exhibit significant relaxation of magnetic interactions at room temperature. This confirms a superparamagnetic behaviour which is manifested by the doublets in the Mössbauer spectra.

Each room temperature Mössbauer spectrum was evaluated using two doublets whose hyperfine parameters are shown in Fig. 2. We assume that the doublet D2 might represent goethite which usually forms sextets at room temperature while $\mathrm{D} 1$ is most probably ferrihydrite. However, their unambiguous identification is dubious because of close similarity of the hyperfine parameters. In addition, in the case of crystal sizes less than $15 \mathrm{~nm}$, both ferrihydrite and goethite may appear as doublets even at $80 \mathrm{~K} \mathrm{[8]}$ as shown for the sample (iii) in Fig. 1b. It is noteworthy that the remaining Mössbauer spectra taken at $80 \mathrm{~K}$ exhibit the same doublet-like features. As far as relative contribution of the doublets is concerned, at $300 \mathrm{~K}$ the area of D2 rises at the expense of D1 from $27 \%$ and $33 \%$ to $56 \%$ for the sample (i), (ii), and (iii), respectively. Similar situation is encountered at $80 \mathrm{~K}$ where the relative area of D2 rises from $29 \%$ and $33 \%$ to $57 \%$.

The Mössbauer spectra of all three samples recorded at $5 \mathrm{~K}$ demonstrate prominent magnetically split patterns as seen in Fig. 3 where the spectrum of sample (iii) is

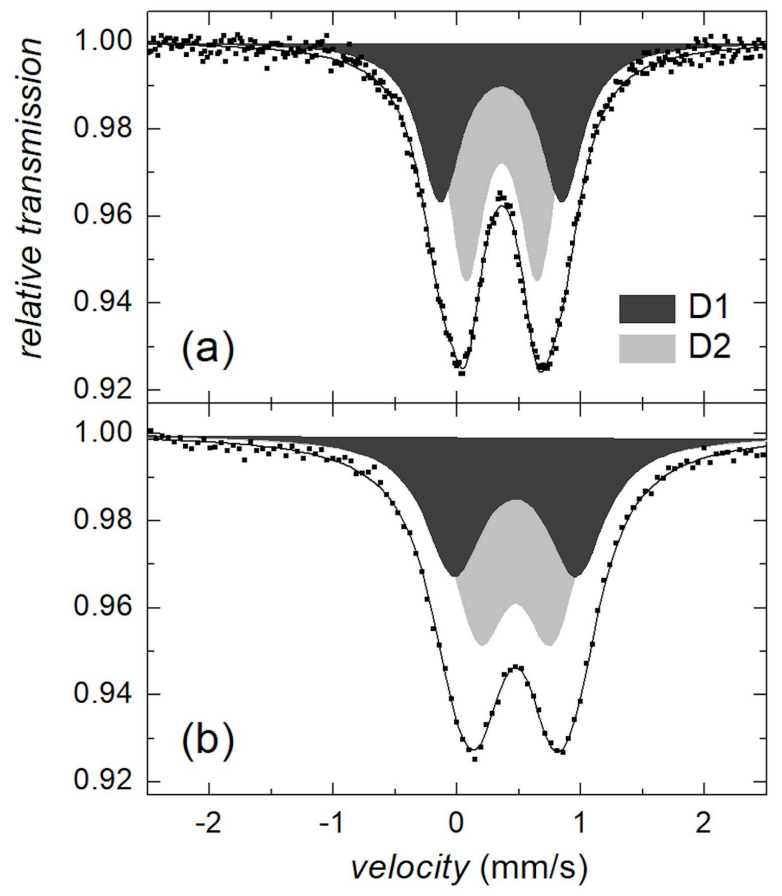

Fig. 1. Mössbauer spectra of sample (iii) measured at $300 \mathrm{~K}(\mathrm{a})$ and at $80 \mathrm{~K}$ (b). Individual spectral components (see the legend) are also shown.

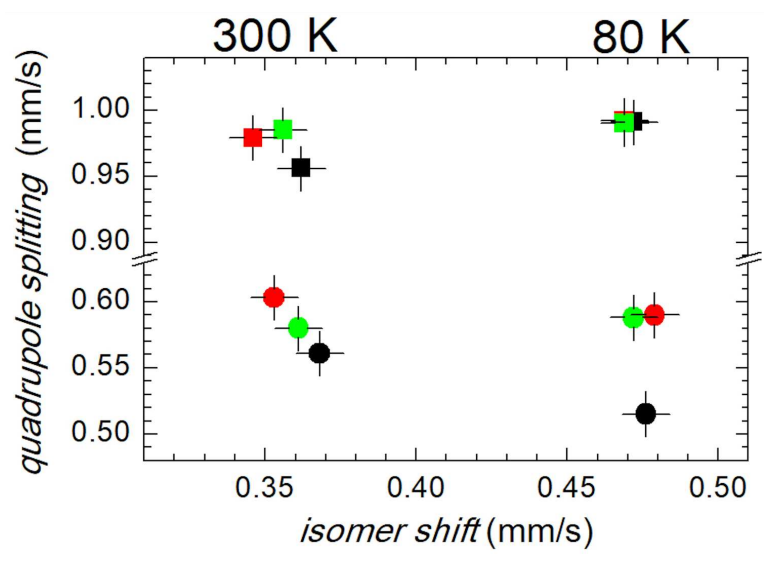

Fig. 2. Hyperfine parameters (quadrupole splitting and isomer shift) of doublets D1 and D2 of samples (i), (ii) and (iii) measured at $300 \mathrm{~K}$ and at $80 \mathrm{~K}$. Squares represent doublet D1 and circles represent doublet D2. Individual samples are coded with colours: black sample (i), red — sample (ii) and green — sample (iii).

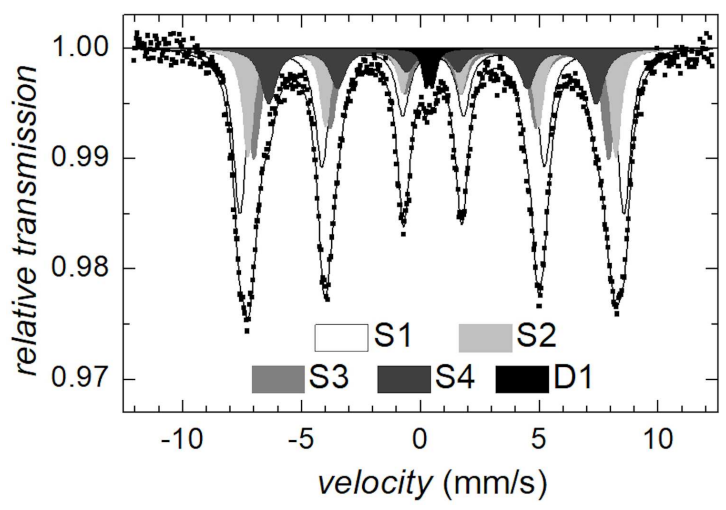

Fig. 3. Mössbauer spectrum of the sample (iii) measured at $5 \mathrm{~K}$. Individual spectral components (see the legend) are also shown.

shown as an example. They were evaluated by one doublet (D) and four sextets (S1-S4). The obtained spectral parameters comprising of relative area $A$, isomer shift IS, quadrupole splitting/shift QS, hyperfine magnetic field $B_{h f}$, and line width $\Gamma$ are listed in Table I. The estimated experimental error margins are of $\pm 1 \%, \pm 0.02 \mathrm{~mm} / \mathrm{s}$, $\pm 0.04 \mathrm{~mm} / \mathrm{s}, \pm 0.17 \mathrm{~T}, \pm 0.04 \mathrm{~mm} / \mathrm{s}$, respectively.

We assign the sextet $\mathrm{S} 1$ to goethite. Slightly different values of the hyperfine parameters are probably due to poor microcrystallinity and/or substitutional effects [8] as evidenced also by considerably high line width. Substitutions by other elements might be responsible namely for small increase in isomer shift. The sextet S2 can be considered to belong to akaganeite which is frequently formed in chlorine-rich environment [9]. The remaining two sextets, S3 and S4, can be assigned to ferrihydrite [10] and lepidocrocite, respectively. Note that due to very small grain sizes as well as poor crystallinity, 
TABLE I

Parameters derived from the Mössbauer spectra of samples (i), (ii) and (iii) that were recorded at $5 \mathrm{~K}$ including relative area $A$, isomer shift IS, quadrupole splitting/shift QS, hyperfine magnetic field of the sextet components $B_{h f}$, and linewidth $\Gamma$

\begin{tabular}{|c|c|c|c|c|c|}
\hline Component & $\begin{array}{c}A \\
\%]\end{array}$ & $\begin{array}{c}\mathrm{IS} \\
{[\mathrm{mm} / \mathrm{s}]}\end{array}$ & $\begin{array}{c}\mathrm{QS} \\
{[\mathrm{mm} / \mathrm{s}]}\end{array}$ & $\begin{array}{l}B_{h f} \\
{[\mathrm{~T}]}\end{array}$ & $\begin{array}{c}\Gamma \\
{[\mathrm{mm} / \mathrm{s}}\end{array}$ \\
\hline \multicolumn{6}{|l|}{ (i) } \\
\hline S1 & 19 & 0.510 & \multirow[b]{5}{*}{0.31} & 50.76 & 0.53 \\
\hline $\mathrm{S} 2$ & 35 & 0.490 & & 48.62 & 0.61 \\
\hline S3 & 33 & 0.497 & & 46.24 & 0.64 \\
\hline $\mathrm{S} 4$ & 12 & 0.512 & & 42.97 & 0.52 \\
\hline $\mathrm{D}$ & 1 & 0.36 & & & 0.32 \\
\hline \multicolumn{6}{|l|}{ (ii) } \\
\hline S1 & 18 & 0.524 & \multirow[t]{2}{*}{-0.036} & 50.47 & 0.65 \\
\hline $\mathrm{S} 2$ & 37 & 0.490 & & 48.53 & 0.68 \\
\hline S3 & 29 & 0.48 & \multirow[t]{2}{*}{-0.01} & 46.13 & 0.62 \\
\hline S4 & 15 & 0.51 & & 42.89 & 0.67 \\
\hline D & 1 & 0.36 & 0.31 & & 0.32 \\
\hline \multicolumn{6}{|l|}{ (iii) } \\
\hline $\mathrm{S} 1$ & 39 & 0.507 & \multirow[t]{2}{*}{-0.051} & 50.17 & 0.67 \\
\hline $\mathrm{S} 2$ & 19 & 0.480 & & 48.06 & 0.51 \\
\hline S3 & 25 & 0.49 & \multirow[t]{2}{*}{-0.081} & 46.33 & 0.65 \\
\hline S4 & 15 & 0.505 & & 42.77 & 0.76 \\
\hline $\mathrm{D}$ & 2 & 0.37 & 0.31 & & 0.34 \\
\hline
\end{tabular}

isomorphous substitutions can be encountered in these natural samples which prevent unambiguous assignment of the individual spectral components. Sizes of fresh precipitates, deposited from seepage waters of complex-ore mine-tailing impoundment at Zlaté Hory, Czech Republic were reported to be of $2-6 \mathrm{~nm}$ [10]. The presented Mössbauer parameters at $5 \mathrm{~K}$ are similar to ours but only one sextet, which was assigned to natural ferrihydrite and nanocrystals of goethite, was used. Nevertheless, it is noteworthy that the relative contribution of S1 significantly increases after bioleaching with the fungus Aspergillus niger. This is at the expense of S2 and S3 which implies a sort of biotransformation of some ironcontaining phases into more stable goethite.

\section{Conclusions}

The goal of this work was to study magnetic phases of iron in natural ochres by the Mössbauer spectrometry. Particles with non-magnetic behaviour were observed at room temperature. They, however, exhibit magnetic behaviour at temperature of $5 \mathrm{~K}$. Occurrence of goethite mineral phase was proved. The hyperfine parameters derived from low-temperature Mössbauer spectra indicate presence of additional magnetic phases. They resemble the parameters of those which are similar to akaganeite, lepidocrocite, and/or ferrihydrite. Nevertheless, rather high degree of structural imperfections, as well as possible substitutional effects are responsible for deviations in the hyperfine parameters.

After bioleaching with the fungus Aspergillus niger the investigated natural ochre exhibits transformation of some iron-containing phases, most probably that of akaganeite and ferrihydrite, into goethite which belongs to the most stable iron hydroxides.

\section{Acknowledgments}

This work was supported by the grants VEGA $1 / 0164 / 17,1 / 0130 / 20$, by the European Regional Development Fund-Project "Centre for Advanced Applied Sciences" No. CZ.02.1.01/0.0/0.0/16_019/0000778 and by the grant of the Comenius University UK/61/2019.

\section{References}

[1] J. Čurlík, L. Jurkovič, Pedogeochémia, Univerzita Komenského v Bratislave, 2012.

[2] A. Kingery-Schwartz, R.S. Popelka-Filcoff, D.A. Lopez, F. Pottier, P. Hill, M.D. Glascock, Open J. Archaeom. 1, 72 (2013).

[3] L. Čanecká, M. Bujdoš, M. Gregor, P. Hudec, K. Boriová, J. Dudová, Separat. Sci. Technol. 49, 721 (2014).

[4] M. Kolenčík, M. Urík, J. Štubňa, Chem. Listy 108, 1040 (2014)

[5] P. Gütlich, E. Bill, A.X. Trautwein, Mössbauer Spectroscopy and Transition Metal Chemistry - Fundamentals and Applications, Springer, Germany 2011.

[6] M. Urík, F. Polák, M. Bujdoš, M.B. Miglierini, B. Milová-Žiaková, B. Farkas, Z. Goneková, H. Vojtková, P. Matúš, Sci. Tot. Environm. 664, 683 (2019).

[7] T. Žák, Y. Jirásková, Surf. Interface Anal. 38, 710 (2006).

[8] R.E. Vandenberghe, E. De Grave, C. Landuydt, L.H. Bowen, Hyperf. Interact. 53, 176 (1990).

[9] R.M. Cornell, U. Schwertmann, The Iron Oxides, Wiley-VCH, Weinheim 2003.

[10] J. Filip, R. Zboril, O. Schneeweiss, J. Zeman, M. Cernik, P. Kvapil, M. Otyepka, Environm. Sci. Technol. 41, 4367 (2007). 